Document downloaded from:

http://hdl.handle.net/10251/63864

This paper must be cited as:

González Pedro, MV.; González-Pedro, V.; Xu, X.; Mora-Sero, I.; Bisquert Mascarell, JA. (2010). Modeling High-Efficiency Quantum Dot Sensitized Solar Cells. ACS Nano. 4(10):5783-5790. doi:10.1021/nn101534y.

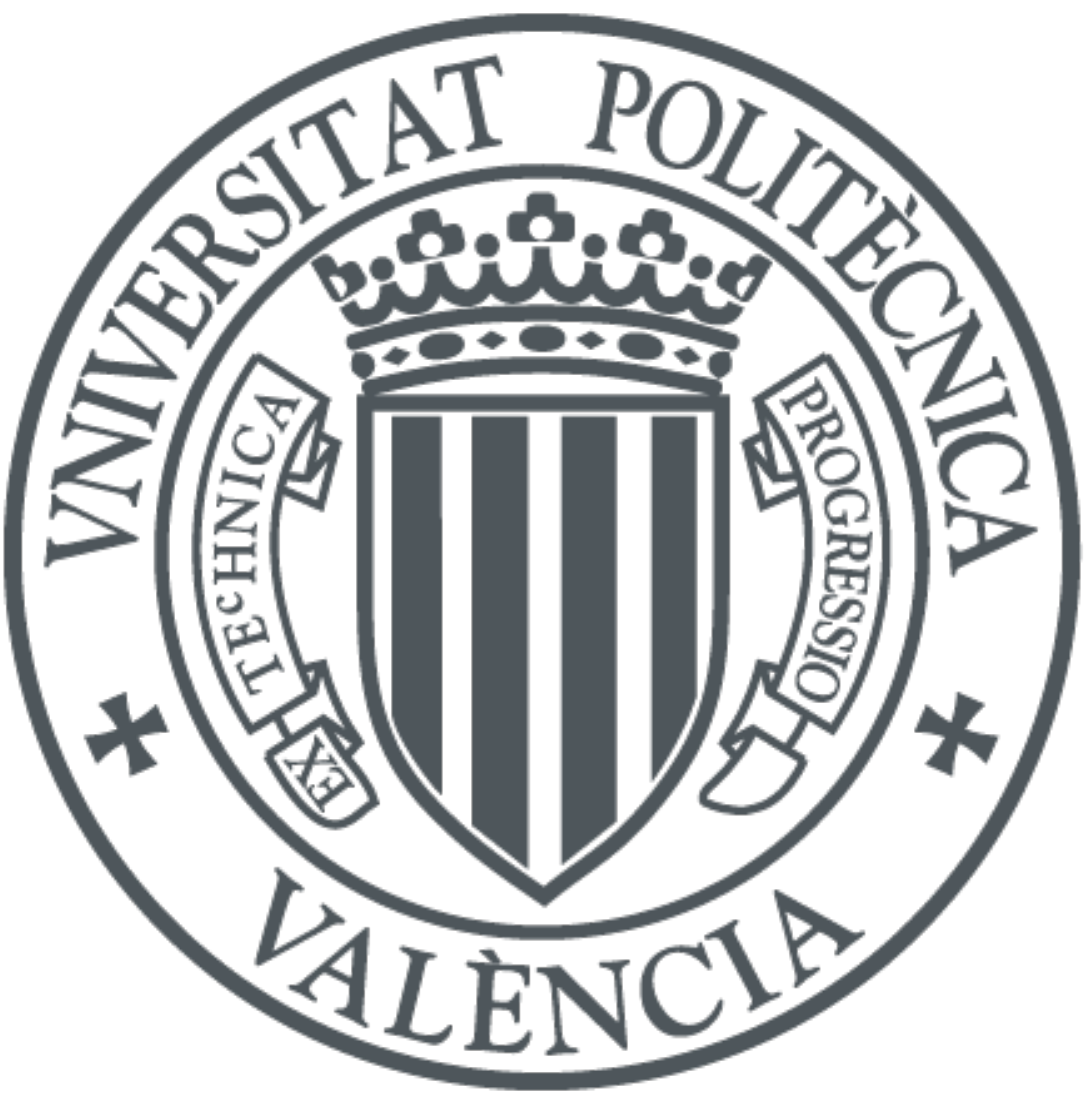

The final publication is available at

http://dx.doi.org/10.1021/nn101534y

Copyright American Chemical Society

Additional Information 


\title{
Modeling High-Efficiency Quantum Dot Sensitized Solar Cells
}

\author{
Victoria González-Pedro, Xueqing Xu, Iván Mora-Seró,* and Juan Bisquert \\ Photovoltaic and Optoelectronic Devices Group, Departament de Física, Universitat Jaume I, 12071 Castelló, Spain. ${ }^{\ddagger}$ Current address: Guangzhou Institute of Energy \\ Conversions, CAS, 510640 Guangzhou, P.R. China.
}

I norganic semiconductors constitute promising materials for the development of sensitized solar cells, where an extremely thin semiconductor layer, sandwiched between two wide band gap semiconductors, acts as absorber layer, ${ }^{1,2}$ or in the same configuration than in dye sensitized solar cells (DSCs) ${ }^{3}$ but using instead of molecular dyes the inorganic semiconductor as light absorbing material. In this last case semiconductor materials are commonly used in the form of quantum dots $(\mathrm{QDs})^{4}$ giving place to quantum dot sensitized solar cells (QDSCs). The use of inorganic semiconductor QDs presents many attractive features for the development of photovoltaic devices. They increase the robustness of the cell and can be prepared in a relatively simple way, with a tunable band gap from the infrared to the ultraviolet region by the control of QD composition and size, ${ }^{5}$ also maintaining beneficial properties of the bulk semiconductors as high molar extinction coefficient ${ }^{5}$ and large intrinsic dipole moments. ${ }^{6,7}$ In addition, the interactions between semiconductor QDs and other compounds can be also advantageous in nanocomposites with enhanced light collecting properties, expanding the light wavelength absorption range $\mathrm{e}^{8,9}$ and/or reducing the overall recombination in the nanocomposite supracollector. ${ }^{10,11}$

Although the conversion efficiency, $\eta$, of QDSCs currently lags significantly behind maximum efficiencies obtained for DSCs, the gap has been quickly reduced in the past few years and efficiencies close to $4 \%$ under full 1 sun illumination have been reported. ${ }^{12,13}$ This fact is significant because the optimal QDSCs configuration has not been obtained yet, and issues as the opti-
ABSTRACT With energy conversion efficiencies in continuous growth, quantum dot sensitized solar cells (QDSCS) are currently under an increasing interest, but there is an absence of a complete model for these devices. Here, we compile the latest developments in this kind of cells in order to attain high efficiency QDSCs, modeling the performance. $\mathrm{CdSe} Q D$ s have been grown directly on a $\mathrm{TiO}_{2}$ surface by successive ionic layer adsorption and reaction to ensure high QD loading. ZnS coating and previous growth of CdS were analyzed. Polysulfide electrolyte and $\mathrm{Cu}_{2} \mathrm{~S}$ counterelectrodes were used to provide higher photocurrents and fill factors, FF. Incident photon-tocurrent efficiency peaks as high as $82 \%$, under full 1 sun illumination, were obtained, which practically overcomes the photocurrent limitation commonly observed in QDSCs. High power conversion efficiency of up to $3.84 \%$ under full 1 sun illumination $\left(V_{\mathrm{oc}}=0.538 \mathrm{~V}, j_{\mathrm{sc}}=13.9 \mathrm{~mA} / \mathrm{cm}^{2}, \mathrm{FF}=0.51\right)$ and the characterization and modeling carried out indicate that recombination has to be overcome for further improvement of QDSC.

KEYWORDS: sensitized solar cells - quantum dots $\cdot$ cadmium selenide $\cdot$ cadmium sulphide · titanium dioxide · SILAR · impedance spectroscopy

cal absorption, charge recombination, hole scavenging, electrolyte, recombination, and series resistance (counterelectrode effect) need to be improved. ${ }^{14,15}$ So far research in this field has focused mainly on showing the validity of the concept and increasing efficiencies and photocurrents of these cells, which were rather low in the beginning. Different semiconductor materials, semiconductor preparation, attaching modes, surface treatments, electrolyte configurations, counter-electrodes, to cite some examples, have been checked in order to improve the performance of QDSCs, but there are few examples of a complete characterization and modeling of photovoltaic devices, ${ }^{14,16,17}$ beyond the conventional current-potential $(J-V)$ curves and incident photon-to-current efficiency (IPCE) measurements. As a consequence no clear model and characterization method have been developed to identify unambiguously the limiting factors in these devices and the real effect in the cell performance of each
*Address correspondence to
sero@fca.uji.es. 

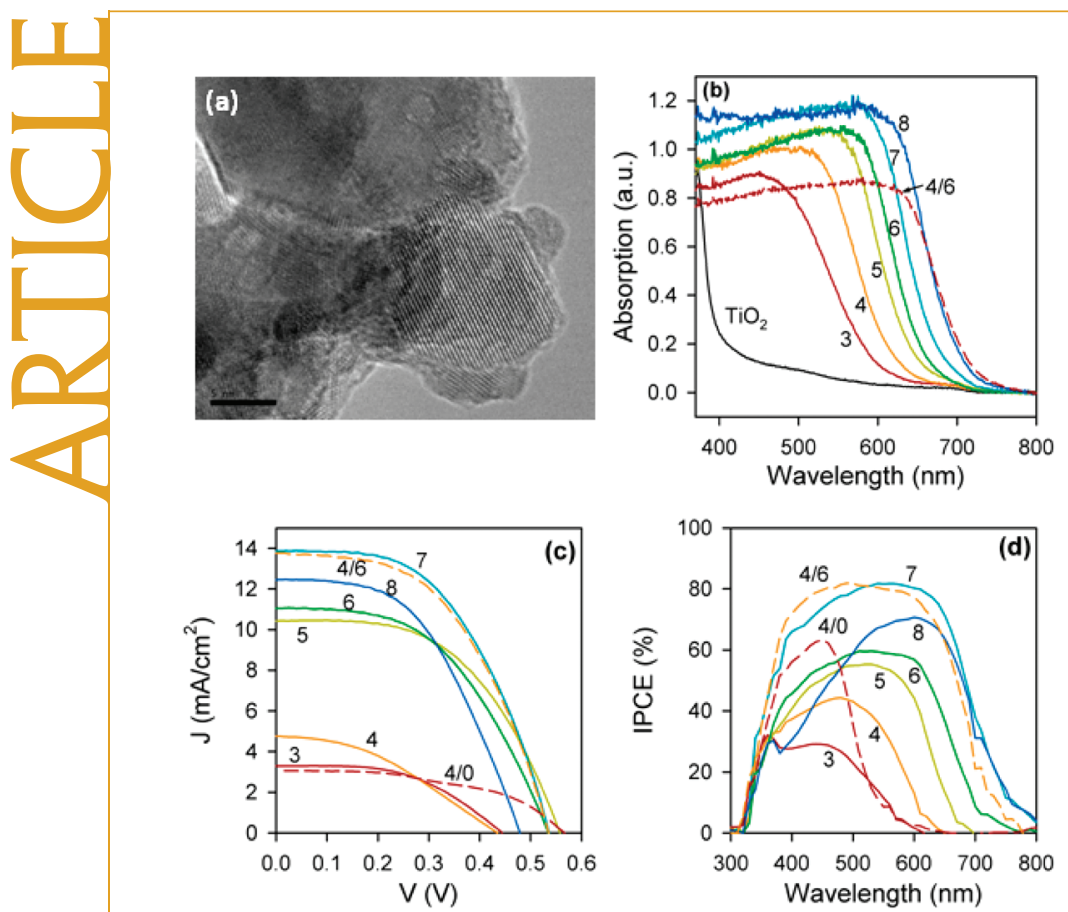

Figure 1. Photovoltaic performance of QDSCs: (a) high-resolution TEM image of CdSe-coated electrode using seven SILAR cycles; (b) absorption spectra of QD sensitized electrodes; (c) $J-V$ curves and (d) IPCE of QDSCs. All the graphics show the behavior depending on the number SILAR cycles. Single number in the legend represents the number of CdSe SILAR cycles. The notation $X / Y$ is referred to $\mathrm{CdS} / \mathrm{CdSe}$ samples where $X$ and $Y$ are the number of CdS and CdSe SILAR cycles, respectively. The expected $j_{\mathrm{sc}}$ for each cell according to the integration of the IPCE curve and the solar irradiance has been compared with the measured $j_{\mathrm{sc}}$, obtaining variations lower than $8 \%$.

of the modifications that have been explored.

Here we show that by compiling the current developments in QDSCs it is possible to overcome the commonly observed photocurrent limitation in these devices. CdSe QDs have been grown directly on a $\mathrm{TiO}_{2}$ surface by successive ionic layer adsorption and reaction (SILAR) ${ }^{16}$ to ensure high QD loading. ZnS coating ${ }^{14,18,19}$ and previous growth of $\mathrm{CdS}^{20}$ were analyzed. Polysulfide electrolyte ${ }^{12,20}$ and $\mathrm{Cu}_{2} \mathrm{~S}$ counterelectrodes $^{18,21}$ were used to provide higher photocurrents and fill factors, FF. IPCE peaks as high as $82 \%$, under full 1 sun illumination, were obtained, which practically overcomes the photocurrent limitation commonly observed in QDSCs. High power conversion efficiency of up to $3.84 \%$ under full 1 sun illumination $\left(V_{\text {oc }}=0.538 \mathrm{~V}, j_{\mathrm{sc}}=13.9 \mathrm{~mA} / \mathrm{cm}^{2}, \mathrm{FF}=0.51\right)$ was obtained. In addition, a careful optoelectronic characterization of the device performance allowed us to model the characteristic features of QDSCs in comparison with the conventional DSCs, showing that there are fundamental differences between the recombination processes of these two cells, as it has been previously suggested ${ }^{15}$ and is demonstrated in this work.

\section{RESULTS AND DISCUSSION}

In the development of QDSCs two main approximations for the preparation of high harvesting material have been employed: the use of presynthesized colloi-

\begin{tabular}{|c|c|c|c|c|c|c|}
\hline $\begin{array}{l}\text { number of SILAR } \\
\text { cycles CdS/CdSe }\end{array}$ & $V_{\text {oc }}(\mathrm{V})$ & $j_{s c}\left(\mathrm{~mA} / \mathrm{cm}^{2}\right)$ & FF & $\eta(\%)$ & $\Delta E_{\mathrm{CB}-\mathrm{TiO}_{2}}(\mathrm{eV})$ & $\beta$ \\
\hline $0 / 3$ & 0.451 & 3.3 & 0.49 & 0.73 & 0 & 0.44 \\
\hline $0 / 4$ & 0.301 & 5.0 & 0.42 & 0.63 & 0.03 & \\
\hline $0 / 5$ & 0.573 & 10.3 & 0.53 & 3.13 & 0.03 & 0.45 \\
\hline $0 / 6$ & 0.550 & 11.3 & 0.48 & 2.98 & 0.05 & 0.39 \\
\hline $0 / 7$ & 0.538 & 13.9 & 0.51 & 3.83 & 0.16 & 0.32 \\
\hline $0 / 8$ & 0.479 & 12.6 & 0.51 & 3.09 & 0.16 & 0.31 \\
\hline $4 / 6$ & 0.548 & 13.8 & 0.50 & 3.78 & 0.125 & 0.29 \\
\hline $4 / 0$ & 0.569 & 3.06 & 0.48 & 0.84 & 0.03 & 0.27 \\
\hline
\end{tabular}

${ }^{a}$ Photovoltaic properties of the manufactured QDSCs, photocurrent $j_{s c,}$ open circuit voltage $V_{\text {oc }}$, fill factor $F F$, and efficiency $\eta$, as a function of the different surface modi-

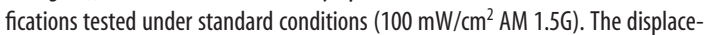
ment of the $\mathrm{TiO}_{2} \mathrm{CB}$ observed by IS (see Figure $2 \mathrm{a}, \mathrm{b}$ ) and $\beta$ parameter obtained from $\beta$-recombination model ${ }^{27,28}$ are also included.

dal QDs ${ }^{17,18}$ and the direct growth of QDs on a $\mathrm{TiO}_{2}$ surface by chemical bath deposition (CBD) ${ }^{19,20}$ or SILAR. ${ }^{16}$ Colloidal QDs present good performance but low QD loading and CBD higher loading but can present QD aggregation, ${ }^{14}$ which introduces a deleterious effect in cell performance. ${ }^{15}$ SILAR offers the possibility of high QD loading with low aggregation (see Figure 1a and Supporting Information section S1). To prepare the QD$\mathrm{SCs}, \mathrm{TiO}_{2}$ nanostructured electrodes with two different layers have been employed, one with small $\mathrm{TiO}_{2}$ particles to ensure high QD loading and another one with bigger particles to increase the light scattering and consequently the light harvesting. After SILAR sensitization, sensitized electrodes present a uniform distribution of QDs along the sample's cross section (see Supporting Information section S2).

The absolute absorption of the sensitized samples increases with the number of SILAR cycles (Figure 1b). In addition the absorption onset shifts to longer wavelengths with the number of SILAR cycles indicating that (i) a reduction of the quantum confinement ${ }^{5}$ occurs and (ii) for the thicker deposits the increased absorption near the bandgap, with smaller absorption coefficient, also contributes to the red shift. ${ }^{15}$ QDSCs with different CdSe SILAR cycles were prepared and characterized with $J-V$ curves (see Figure 1c and Table 1). All the samples were coated with $\mathrm{ZnS}$ to reduce the recombination of electrons in the $\mathrm{TiO}_{2}$ to the polysulfide electrolyte ${ }^{14,19}$ with a consequent increase in the photocurrent. On the other hand, $\mathrm{Cu}_{2} \mathrm{O}$ was used as counter-electrode material to enhance the charge transfer between counter-electrode and polysulfide, ${ }^{18,21}$ reducing the series resistance of the device and increasing the FF to a value of $\sim 0.5$ (see Table 1 ), significantly higher than the reported values of $0.3-0.4$ using Pt or Au as counter-electrodes. ${ }^{18}$ The combination of these strategies allow us to obtain high QDSCs with a maximum efficiency of $\eta=3.84 \%$ under full 1 sun illumination for seven CdSe SILAR cycles, using mask and no antireflective layer. The IPCE of the prepared samples (Figure 1d) increases with the number or SILAR cycles 
and the onset shifts to longer wavelengths, in good correlation with absorption measurements (Figure 1b). This trend is broken for a number of cycles higher than seven, and a reduction of IPCE and cell performance is observed for eight cycles (see Figure 1). For seven cycles an IPCE peak as high as $82 \%$ was obtained, which is one of the highest reported IPCE for this kind of cells.

To establish a model of QDSCs performance impedance spectroscopy (IS) measurements were carried out (Figure 2a,b). IS spectra were analyzed using an analogous model as that employed in DSC characterization ${ }^{22}$ (Figure $2 \mathrm{c}$ ). It is important to remark that the equivalent circuit presented in Figure $2 \mathrm{c}$ reflects the physical processes of carrier transport, charge transfer, and recombination that take place in the device. The equivalent circuit elements have the following physical meaning: ${ }^{22} C_{\mu}\left(=C_{\mu} \cdot L\right.$, where $L$ is the $\mathrm{TiO}_{2}$ layer thickness) is the chemical capacitance that stands for the change of electron density as a function of the Fermi level, and it monitors the distribution of traps states in the bandgap of the $\mathrm{TiO}_{2}$ semiconductor. $R_{\mathrm{tr}}\left(=r_{\mathrm{tr}} \cdot L\right)$ is the electron transport resistance in the $\mathrm{TiO}_{2}$, directly related to the reciprocal conductivity. $R_{r}\left(=r_{r} \cdot L\right)$ is the recombination resistance, a charge-transfer resistance related to recombination of electrons at the $\mathrm{TiO}_{2} /$ electrolyte interface. $R_{\mathrm{r}}$ is inversely proportional to both the recombination rate and the density of electrons in $\mathrm{TiO}_{2} . R_{\mathrm{s}}$ is a series resistance accounting for the transport resistance of the transparent conducting oxide and the connection setup. $Z_{d}$ is the diffusion impedance of the redox species in the electrolyte (we have not considered this effect in this paper). $R_{\text {CE }}$ represents the charge transfer resistance at the counter-electrode/ electrolyte interface. $C_{\mathrm{CE}}$ is the interfacial capacitance at the counter-electrode/electrolyte interface. The first three mentioned elements $\left(C_{\mu}, R_{\mathrm{tr}}\right.$ and $\left.R_{\mathrm{r}}\right)$ are denoted in lowercase letters in Figure $2 \mathrm{c}$ meaning the element per unit length for a film of thickness $L$, because they are distributed in a repetitive arrangement of a transmission line. The physical meaning of this network corresponds to the impedance of diffusion and recombination. ${ }^{23}$ The IS model presented allows an easy correlation between the impedance spectra and the physical processes, permitting to distinguish separately each effect.

The obtained IS spectra (Figure 2a,b) present the Warburg behavior characteristic of a transmission line ${ }^{23}$ (straight line at frequencies higher than the recombination arc), allowing us to obtain transport parameters of electrons in the $\mathrm{TiO}_{2}$. The decrease of the recombination resistance, $R_{\mathrm{r}}$, with the applied voltage can be also visualized as the decrease of the semicircle radius. Using
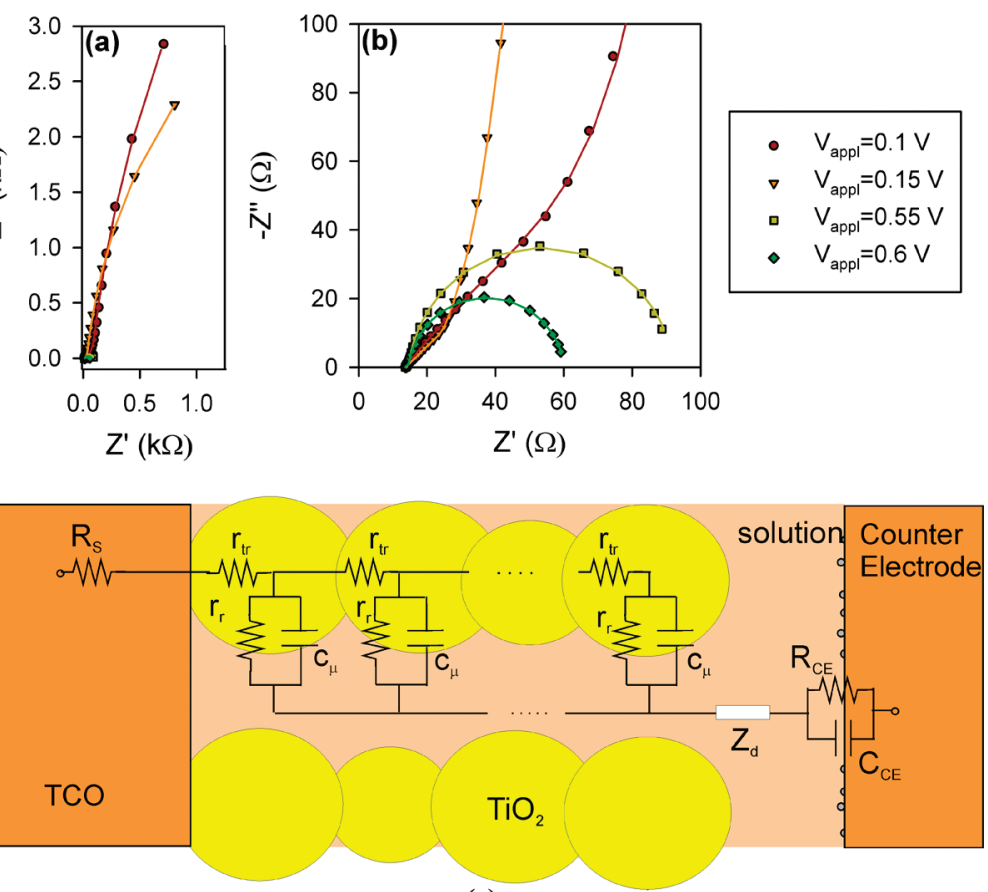

(c)

Figure 2. (a) Impedance measurement of QDSCs (symbols) using seven CdSe SILAR cycles and $\mathrm{ZnS}$ coating at different applied forward bias, $V_{\text {appl, }}$ under dark conditions. (b) Zoom of graph a. Transmission line pattern, observed as a straight line in the high frequency range, is clearly observed for $V_{\text {appl }}=0.1$ and $0.15 \mathrm{~V}$. Solid lines correspond to the fit using (c) an analogous equivalent circuit as the one employed in $\mathrm{SCs}^{22}$ but considering a short circuit in the electrolyte, $Z_{\mathrm{d}}=0 \Omega$.

polysulfide electrolyte commonly prevents the observation of the Warburg behavior due to the high transfer resistance with the Pt counter-electrode, ${ }^{14}$ because the arc due to the counter-electrode at high frequencies is large enough to hide this behavior, but the use of $\mathrm{Cu}_{2} \mathrm{~S}$ counter-electrode reduces dramatically these charge transfer resistances (Figure $3 \mathrm{~b}$ ). Figure $3 \mathrm{~b}$ shows the dramatic effect in the charge transfer resistance between the counter-electrode and the polysulfide electrolyte when $\mathrm{Cu}_{2} \mathrm{O}$ is used instead of $\mathrm{Au}$ or Pt counterelectrodes. Au and Pt counter-electrodes have been checked with electrodes sensitized with CdSe QDs grown by chemical bath deposition (CBD). We have previously shown that the material of the counterelectrode does not affect the parameters of the sensitized electrode, ${ }^{14}$ and vice versa, so the preparation process of the QD does not affect the counter-electrode transfer resistance. On the other hand, the series resistance (Figure $3 \mathrm{a}$ ) is independent of the applied voltage as is expected; its values range from 2 to $3.5 \Omega \cdot \mathrm{cm}^{2}$ for all the samples except for the one prepared with 3 SILAR. These values are comparable with the values obtained for good performing DSCs. In this case of 3 SILAR cycles the higher $R_{\mathrm{s}}$ is produced because this sample was measured without a metallic contact soldered onto the FTO surface.

The observation of the transmission line in the impedance spectra permits a complete characterization of the QDSCs and also allows us to obtain different cell 

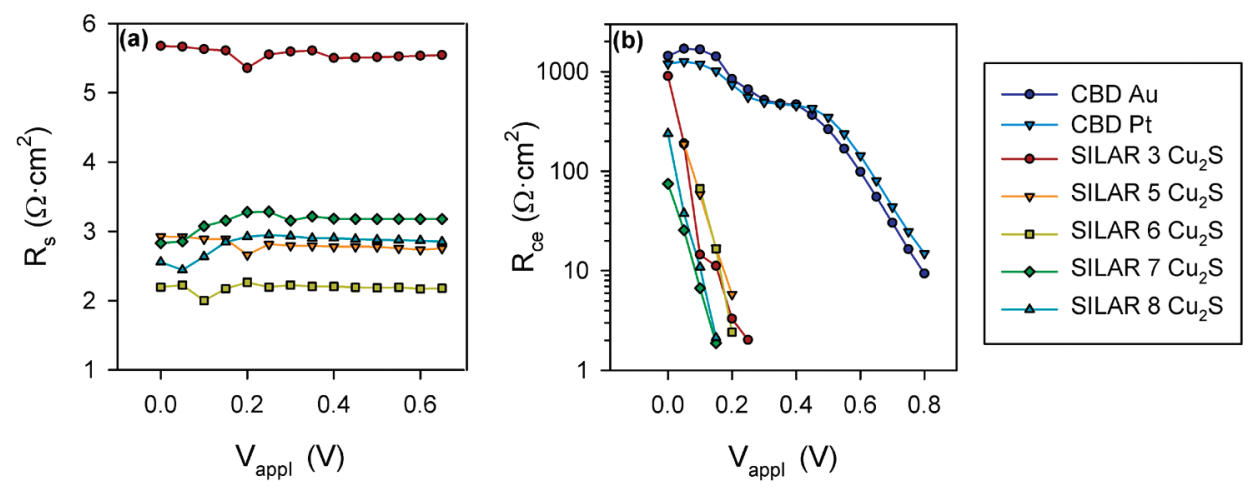

Figure 3. (a) Series resistance, $R_{\mathrm{s}}$ and (b) counter-electrode resistance, $R_{\mathrm{ce}}$, as function of applied forward bias, $V_{\mathrm{appl}}$, of solar cells with different number of CdSe SILAR cycles and ZnS coating.

parameters as a function of the voltage drop in the sensitized electrode $V_{\mathrm{F}}$. IS enables extracting the voltage drop in the sensitized electrode, $V_{F}$, at each applied potential, $V_{\text {appl, }}$ by subtracting the effect of the series resistance and counter-electrode charge transfer resistance on both $R_{\mathrm{r}}$ and $C_{\mu}$ as follows: $V_{\mathrm{F}}=V_{\mathrm{appl}}-V_{\mathrm{s}}-V_{\mathrm{ce}}$ where $V_{s}$ and $V_{\text {ce }}$ are the potential drop at the series resistance and at the counter-electrode, respectively. $V_{F}$ is proportional to the rise of the Fermi level of electrons in $\mathrm{TiO}_{2}, V_{\mathrm{F}}$ $=\left(E_{\mathrm{Fn}}-E_{\mathrm{F} 0}\right) / q$, where $q$ is the positive elementary charge and $E_{\mathrm{Fn}}$ and $E_{\mathrm{F} 0}$ are the electron Fermi level and the electron Fermi level at the equilibrium, respectively. To analyze the recombination resistance, $R_{\mathrm{r}}$ on the basis of a similar number of electrons (i.e., the same distance between the electron Fermi level, $E_{\mathrm{Fn}}$, and conduction band (CB) of $\mathrm{TiO}_{2}, E_{\mathrm{CB}}$ ) the shift of instead of $C B$ (indicated for each sample in Table 1) has been corrected in Figure 4, where the voltage scale is $V_{F, \text { shifted. }}$ The criterion for the modified scale is that the chemical capacitances of all the analyzed samples overlap (Figure 4b) because the chemical capacitance ${ }^{24}$ is directly related with the difference $E_{\mathrm{CB}}-E_{\mathrm{Fn}}$, by the relation $C_{\mu} \alpha \exp \left[-\left(E_{\mathrm{CB}}-E_{\mathrm{Fn}}\right) /\left(k_{\mathrm{B}} T\right)\right]$. The same shift applied to the chemical capacitance has been applied to the other parameters shown in Figure 4.

The analysis of the chemical capacitance, $C_{\mu}$ points out to a downward displacement of the $\mathrm{TiO}_{2}$ conduction band (CB) that in-
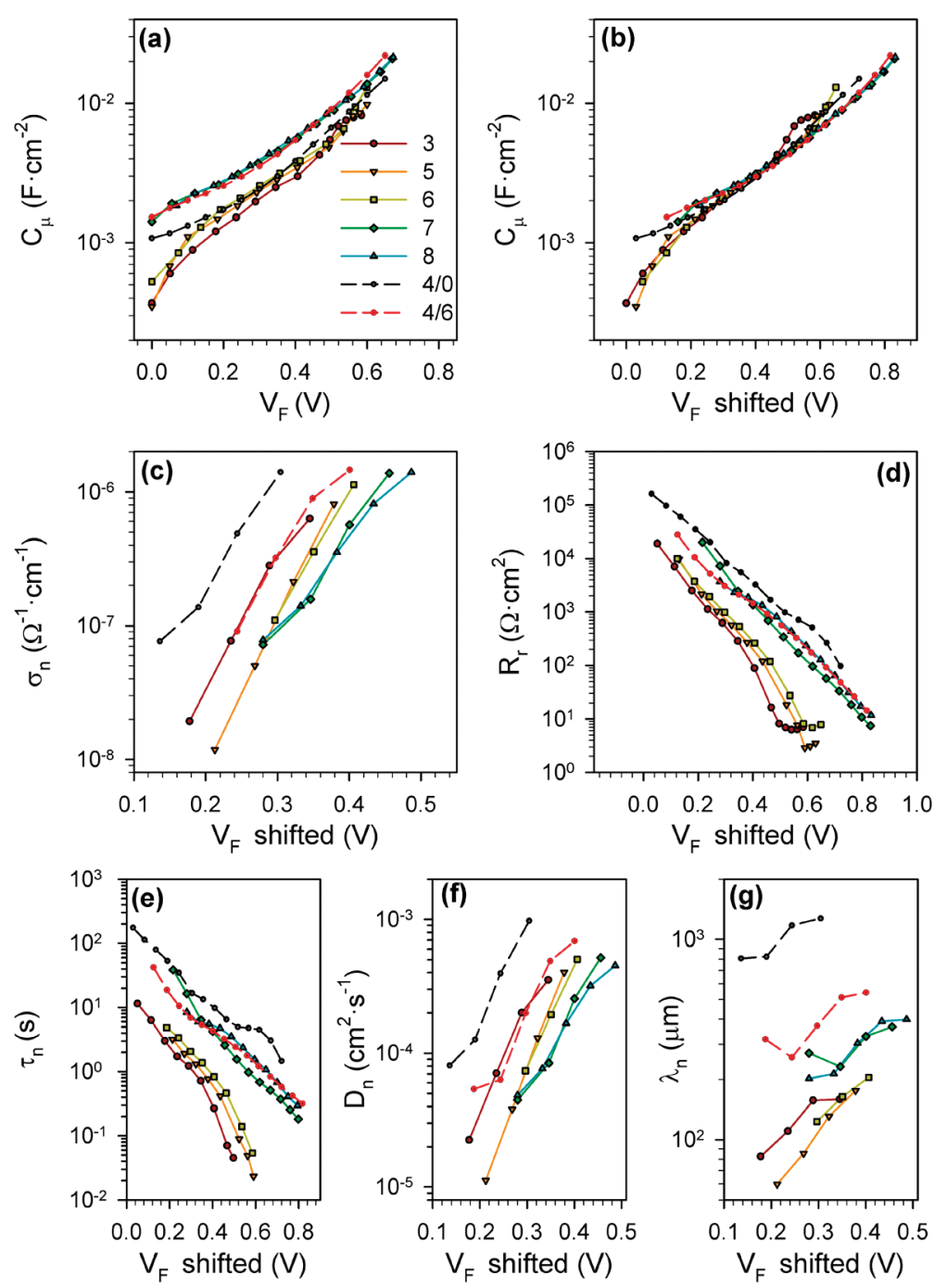

Figure 4. Impedance characterization of QDSCs. Behavior of different parameters of the sensitized electrode obtained by IS, depending on the number SILAR cycles as function of the voltage drop in the electrolyte, $V_{F}$ or $V_{F, \text { shifted }}$ (where the displacement of the $\mathrm{TiO}_{2} \mathrm{CB}$ has been removed). Chemical capacitance, $C_{\mu}$, as function of (a) $V_{F}$ and (b) $V_{F, \text { shifted. }}$ (c) Electron conductivity, $\sigma_{n}$. (d) Recombination resistance $R_{r}$. (e) Electron lifetime, $\tau_{\mathrm{n}}$. (f) Electron diffusion coefficient in $\mathrm{TiO}_{2}, D_{\mathrm{n}}$. (g) Small perturbation diffusion length in $\mathrm{TiO}_{2}, \lambda_{n}$. 
vious report, now, the increasing amount of CdSe with the number of SILAR cycles prevents the direct contact of the polysulfide electrolyte $\left(\mathrm{pH} \approx 12\right.$ ) with $\mathrm{TiO}_{2}$, which decreases the effective "observed" $\mathrm{pH}$ of the $\mathrm{TiO}_{2}$ surface, consequently reducing the $\mathrm{TiO}_{2} \mathrm{CB}$ position. The downward displacement of $\mathrm{CB}$ can be removed by a voltage shift (Figure 4b) as it has been commented before. With this correction cells can be consequently compared in the same conditions of number of electrons in $\mathrm{TiO}_{2}$.

Some authors use the integrated electron density (from the chemical capacitance) to compare the recombination rates, but we believe that using the corrected voltage is a more powerful procedure, as this method allows the simultaneous analysis of the IS and $J-V$ data. A systematic procedure to realize this approach is being developed. ${ }^{26}$ The number of CdSe SILAR cycles affects the recombination resistance, $R_{\mathrm{r}}$ (Figure $4 \mathrm{~d}$ ), with an increase of $R_{\mathrm{r}}$ with the number of cycles due to a higher loading of CdSe QDs that act themselves as a blocking layer. On the other hand, from the transport resistance $R_{\mathrm{tr}}$ it is possible to determine the electron conductivity in the $\mathrm{TiO}_{2}$ as $\sigma_{n}=L / R_{\mathrm{tr}} \cdot S$, where $L$ is the thickness of $\mathrm{TiO}_{2}$ and $S$ is the area. $\sigma_{\mathrm{n}}$ decreases with the number of SILAR cycles (Figure 4c), and this diminution can be attributed to the filling of $\mathrm{TiO}_{2}$ pores. As the pore size decreases with the number of SILAR cycles, the diffusion into the pores of the chemical species in the electrolyte, allowing a good screening, is reduced, affecting $\sigma_{\mathrm{n}}$. Knowing the values of $\mathrm{C}_{\mu}, R_{\mathrm{r}}$, and $R_{\mathrm{tr}}$ makes it possible to calculate important electron parameters in the device, such as lifetime, $\tau_{n}$, diffusion coefficient, $D_{n}$, and the small perturbation diffusion length, $\lambda_{n}{ }^{22}$ see Figure 4 panels e, $d$, and $f$, respectively.

The increase of $R_{\mathrm{r}}$ with the amount of QDs produces an increase of lifetime that is partially compensated with a reduction in the $D_{\mathrm{n}}$ as a consequence of the decrease of $\sigma_{\mathrm{n}}$. Finally the calculated small perturbation diffusion length values are significantly higher than the $\mathrm{TiO}_{2}$ thickness $(\sim 14 \mu \mathrm{m})$, indicating that the prepared QDSCs are not transport limited as is expected in a good performing solar cell. As explained elsewhere, ${ }^{27}$ the small perturbation diffusion length $\lambda_{n}=$ $\left(D_{n} \cdot \tau_{n}\right)^{1 / 2}$ is well-defined, while a general definition of diffusion length is not unambiguous. In the

$\beta$-recombination model ${ }^{27}$ the recombination in DSC is proportional to $n^{\beta}$, where $n$ is the electron density in the $\mathrm{TiO}_{2}$ and $\beta$ is a parameter. A model based on charge transfer via a distribution of surface states on the $\mathrm{TiO}_{2}$ predicts $\beta=1 / 2+T / T_{0}$ where $T$ is the temperature and $T_{0}$ relates to exponential distribution of traps in $\mathrm{TiO}_{2}{ }^{28}$ with a minimum value of $\beta=0.5$. In DSCs $\beta$ has values that commonly lie between $0.5-0.7$. In the $\beta$-recombination model the slope of $\ln \left(R_{\mathrm{r}}\right)$ vs $V_{\mathrm{F}}$ allows the calculation of $\beta$, taking into account the relation: ${ }^{27,28}$ $R_{\mathrm{r}}=R_{0}^{\prime} \exp \left(-q \beta V_{\mathrm{F}} /\left(k_{\mathrm{B}} T\right)\right)$, where $q$ is the positive elemental charge, $T$ is the temperature, $k_{\mathrm{B}}$ is the Boltz-

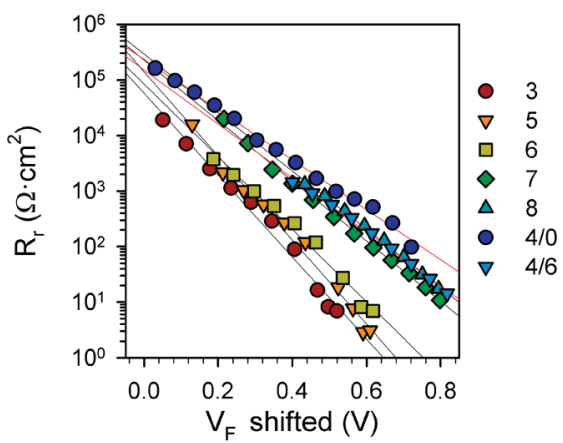

Figure 5. Experimental data (symbols), from Figure 4d, have been fitted to a straight line (solid black line for CdSe and solid red line for $\mathrm{CdS} / \mathrm{CdSe}$ ). A single number in the legend represents the number of CdSe SILAR cycles. The notation $X / Y$ is referred to $C d S / C d S e$ samples where $X$ and $Y$ are the number of CdS and CdSe SILAR cycles, respectively.

mann's constant, and $R_{0}^{\prime}$ is a pre-exponential factor. In DSCs $\beta$ can vary with the applied voltage but in good performing cells $\beta$ is fairly constant in a wide range of $V_{F}$ values, as occurs in the QDSCs prepared in this work, see Figure 5 . We have obtained $\beta$ in this range. The exponential shape of recombination resistance also warrants the use of a diode model to describe the solar cell $J-V$ curves.

In the case of CdSe QDSCs (Figure 5), for a low number of SILAR cycles the obtained value is close to 0.5 (Table 1), but this value diminishes until close to 0.3 , lower than the $\beta$ minimum, for seven and eight cycles. This fact has important implications because it indicates that there is a clear evolution in the recombination mode between electrodes with low and high QD loading and also that QDs play an active role in the recombination process. As the $\mathrm{TiO}_{2}$ surface is becoming covered with the number of SILAR cycles the recombination process changes from a preferential recombination with the electrolyte to a preferential recombination with the QDs.

An additional proof of this conclusion can be obtained by $V_{\text {oc }}$ decay measurements. To record the $V_{\text {oc }}$ decay we have employed two methods in order to increase the voltage of the sample: (i) open circuit voltage decay (OCVD) where the sample was illuminated and the voltage decay was measured after switching off the light, and (ii) applied bias voltage decay (ABVD) where a voltage in the dark is applied and its decay is analyzed in open circuit conditions. Both measurements give similar results for DSCs, if enough short illumination time is employed in OCVD in order to avoid a significant increase of the temperature. ${ }^{29}$ Taking care of this point we have made both measurements in QDSCs and calculated the lifetime $\tau_{n}{ }^{29}$ (Figure 6). There is a good agreement between $\tau_{n}$ obtained by IS and that obtained by ABVD, see inset Figure 6, carried out both under dark conditions. But lower electron life is measured with OCVD. As QDs are the light sensible part of the device, this difference between light and dark measurements indicates that they play an active role in the 


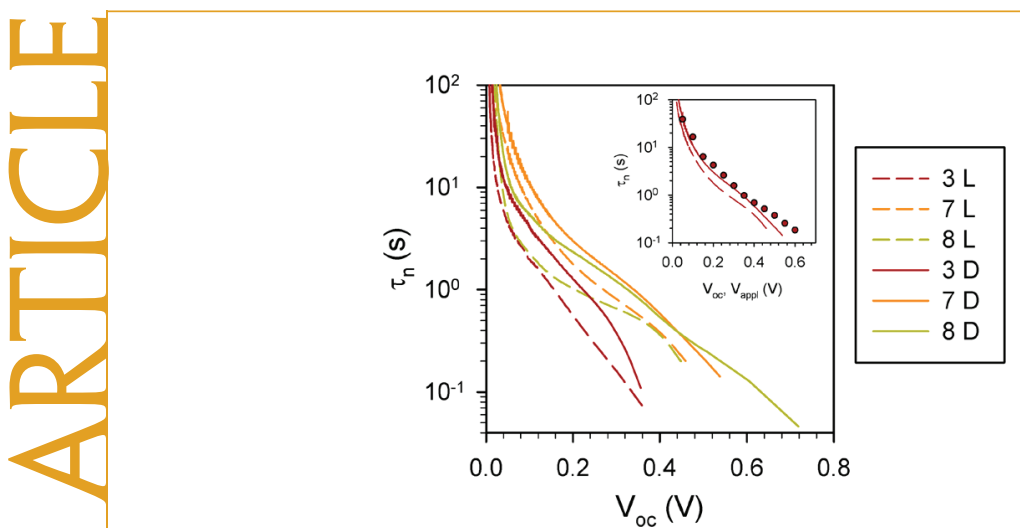

Figure 6. Open circuit voltage decay (OCVD) and applied bias voltage decay (ABVD) characterization of QDSCs. Comparison of the electron lifetime between the photoinduced OCVD (L) and the applied voltage ABVD (D) for QDSCs samples with different number of SILAR cycles. Inset: Comparison between the electron lifetimes extracted from OCVD and IS measurements for the QDSCs prepared with seven SILAR cycles.

recombination process. Illumination produces electron-hole pairs that can be trapped in QD surface states, enhancing the recombination of electrons in the $\mathrm{TiO}_{2}$ with holes in the QDs or be back injected in electron trap states in the QD, producing a recombination pathway that it is practically nonexistent in good performing DSCs.

If QDs present this active role in recombination, there should be expected also an influence of the QD material. To investigate this we have analyzed the use of CdS as sensitizer. This material is specially interesting because it is extremely easy to prepare and also because it has been reported that its combined use with CdSe can enhance cell performance. ${ }^{20,30}$ An example of the QDSCs using CdS is reported in Figure $1 \mathrm{~b}-\mathrm{d}$ (see also Supporting Information S3). CdS presents lower photocurrent than CdSe cells because its band gap is wider (Figure 1c,d). The combined use of CdS and CdSe allows an increase of IPCE in the short wavelength region (Figure 1d) and efficiency close to the maximum obtained for seven CdSe SILAR cycles is obtained with four and six successive SILAR cycles of CdS and CdSe (Figure 1c,d and Table 1).

From the point of view of recombination it is very interesting to note the large difference between $\mathrm{CdS}$ and CdSe sensitized layers. Comparing CdS4 with CdSe3, where the number indicates the number of SILAR cycles, only a slight downward shift of the CdS sensitized $\mathrm{TiO}_{2}$ is observed ( $0.03 \mathrm{eV}$, Table 1 and Figure 3a).
In contrast CdS4 sample presents $R_{r}$ and $\sigma_{\mathrm{n}}$ sensibly higher than $\mathrm{CdSe}$. Both variations go toward an increase of cell performance, indicating that $\mathrm{CdS}$ allows a better cell performance than CdSe, but the cell is limited by the absorption spectral range of CdS. This absorption range can be expanded using $\mathrm{CdSe}$ on top of CdS (see Figure 1d for the case of CdS4/CdSe6). In this sample we have obtained $R_{\mathrm{r}}$ and $\sigma_{\mathrm{n}}$ parameters better than those for $\mathrm{CdSe} 6$ or even $\mathrm{CdSe} 7$ from the point of view of enhanced cell operation. The efficiency obtained for sample CdS4/CdSe6 is very close to the one obtained for CdSe7 (Table 1). A compromise is needed between absorption and pore filling for the combined use of CdS and CdSe, as in the case of CdSe. The use of $\mathrm{CdS}$ increases cell performance in the case of six CdSe SILAR cycles if three or four CdS SILAR cycles are employed, but decreases when five cycles are used (Supporting Information section S4). No further improvement of cell performance has been obtained using $\mathrm{CdS}$ and seven CdSe SILAR cycles. But, the results reported indicate that in a more open structure than the one used in this study the combined use of CdS and CdSe has a higher potential for good performing cells than $\mathrm{CdSe}$ alone provided that the pore filling limitation can be overcome.

A deep analysis of the recombination process in CdS samples points out that cells with only four CdS SILAR cycles present a $\beta$ lower than 0.3 (Supporting Information section S3), indicating that in these conditions the recombination is already preferential with CdS instead of with the electrolyte. We have demonstrated that the type and size of $\mathrm{TiO}_{2}$ covering of QDs plays a fundamental role in the QDSCs recombination; further characterization of cyclic voltammetry under dark conditions also points in the same direction (Supporting Information section S5).

\section{CONCLUSIONS}

We have shown that it is possible to prepare high performing QDSCs that overcome the photocurrent limit, obtaining QDSCs with a record IPCE. We have given a model for the characterization of QDSCs and demonstrated the role of light absorbing QDs in the recombination process. Further increase in QDSC performance will require an increase of $V_{\text {oc }}$ and $\mathrm{FF}$, and to attain this objective a careful control of the recombination through QDs is needed.

\section{MATERIALS AND METHODS}

Preparation on Sensitized $\mathrm{TiO}_{2}$. The electrode configuration was a transparent layer DSL 18NR-T (20 nm average particle size) and a scattering layer WERO-4 (300-400 nm particle size distribution). The FTO $\left(\mathrm{SnO}_{2}: \mathrm{F}\right)$ coated glass was previously covered by a compact layer of $\mathrm{TiO}_{2}$ deposited by spray pyrolisis of titanium(IV)bis(acetoacetonato) di(isopropanoxylate). These electrodes were sintered at $450{ }^{\circ} \mathrm{C}$ for $30 \mathrm{~min}$. The thickness of the mesoporous electrodes was around $14 \mu \mathrm{m}$ (9 $\mu \mathrm{m}$ of transparent layer and $5 \mu \mathrm{m}$ of scattering layer) measured by scanning electron microscopy (SEM) with a JSM-7000F JEOL FEG-SEM system, see Supporting Information section S2.

The mesoporous $\mathrm{TiO}_{2}$ electrodes were in situ sensitized by CdS and CdSe QDs grown by SILAR. For CdS growth the electrodes were successively immersed in two different solutions for 1 min each: one consisting of $0.02 \mathrm{M} \mathrm{Cd}\left(\mathrm{NO}_{3}\right)_{2}$ dissolved in methanol, another of $0.02 \mathrm{M} \mathrm{Na}_{2} \mathrm{~S}$ in methanol/Milli-Q ultrapure 
water (1:1). Following each immersion, rinsing and drying was undertaken using pure methanol and a $\mathrm{N}_{2}$ gun, respectively. All these processes constitute one SILAR cycle. A SILAR of CdSe presents more problems. In order to obtain the Se precursor, we followed the method developed by the group of Grätzel ${ }^{16}, 0.03 \mathrm{M}$ $\mathrm{Cd}\left(\mathrm{NO}_{3}\right)_{2}$ dissolved in ethanol, and another method incorporating the in situ generated $0.03 \mathrm{M} \mathrm{Se}^{2-}$ in ethanol (see ref 16 for more details). For sensitization electrodes were dipped successively in these solutions inside a glovebox under $\mathrm{N}_{2}$ atmosphere. Following each immersion, rinsing and drying was undertaken using pure ethanol and a $\mathrm{N}_{2}$ gun, respectively. In CdS/CdSe samples after $\mathrm{CdS}$ sensitization, electrodes were introduced into the glovebox were the CdSe SILAR cycles were carried out. All the samples analyzed in this study have been coated with $\mathrm{ZnS}$, after SILAR sensitization, by twice dipping alternately into $0.1 \mathrm{M} \mathrm{Zn}\left(\mathrm{CH}_{3} \mathrm{COO}\right)_{2}$ and $0.1 \mathrm{M} \mathrm{Na}_{2} \mathrm{~S}$ solutions for $1 \mathrm{~min} /$ dip, rinsing with Milli-Q ultrapure water between dips. ${ }^{19}$ At least two cells have been prepared at each condition obtaining a variation on cell performance among cells lower than 15\%, for cells not presenting problems during the ensemble process (see an example in Supporting Information section S6).

Solar Cell Configuration. The cells were prepared by assembling the counter-electrode and a QD-sensitized electrode using a scotch spacer (thickness $50 \mu \mathrm{m}$ ) and with a droplet $(10 \mu \mathrm{L})$ of polysulfide electrolyte. Polysulfide electrolyte was $1 \mathrm{M} \mathrm{Na}_{2} \mathrm{~S}, 1 \mathrm{M}$ $\mathrm{S}$, and $0.1 \mathrm{M} \mathrm{NaOH}$ solution in Milli-Q ultrapure water. We have used $\mathrm{Cu}_{2} \mathrm{~S}$ counter-electrodes. The $\mathrm{Cu}_{2} \mathrm{~S}$ counter-electrodes were prepared by immersing brass in $\mathrm{HCl}$ solution at $70{ }^{\circ} \mathrm{C}$ for $5 \mathrm{~min}$ and subsequently dipping it into polysulfide solution for $10 \mathrm{~min}$ resulting in a porous $\mathrm{Cu}_{2} \mathrm{~S}$ electrode. ${ }^{21}$ The area of the cells was $0.24 \mathrm{~cm}^{2}$.

Electrode and Cell Characterization. The absorption spectra were recorded by a Cary 500 UV - vis Varian spectrometer. For TEM measurements a JEM-2100 electron microscope (JEOL) operated at $200 \mathrm{kV}$ was used. The IPCE measurements were performed employing a $150 \mathrm{~W}$ Xe lamp coupled with a monochromator controlled by a computer; the photocurrent was measured using an optical power meter 70310 from Oriel Instruments, using a Si photodiode to calibrate the system. Current-potential curves, cyclic voltammetry, open circuit voltage decay (OCVD) and applied bias voltage decay (ABVD) and impedance spectroscopy measurements were obtained using a FRA-equipped PGSTAT-30 from Autolab. The cells were illuminated using a solar simulator at AM 1.5 G, where the light intensity was adjusted with an NREL-calibrated Si solar cell with a KG-5 filter to 1 sun intensity $\left(100 \mathrm{~mW} \mathrm{~cm}^{-2}\right)$. Impedance spectroscopy measurements were carried out in dark conditions at forward bias, applying a 20 $\mathrm{mV} A C$ sinusoidal signal over the constant applied bias with the frequency ranging between $400 \mathrm{kHz}$ and $0.1 \mathrm{~Hz}$. To verify the stability of the cells during IS measurements $J-V$ curves were performed before and after IS. No significant variation was observed indicating a good stability during the measurement (see an example in Supporting Information section S7). Measurements were carried out using mask and with no antireflective layer. In OCVD measurements samples were illuminated and the decay of $V_{\text {oc }}$ was studied as a function of time after the switch-off of the light. In ABVD a current is forced to cross the device under dark, obtaining an applied voltage, then the current is switched off and the voltage decay is measured in open circuit conditions.

Acknowledgment. This work was supported by the Ministerio de Ciencia e Innovación of Spain under the projects HOPE CSD2007-00007, JES-NANOSOLAR PLE2009-0042, and MAT200762982 , and by Generalitat Valenciana under the project PROMETEO/2009/058. The authors want to acknowledge S. Giménez for the TEM measurements and the calculation of expected currents from IPCE, A. Braga for the preparation of CdS sensitized substrates, and also the SCIC of University Jaume I (Spain) for providing the microscopy facilities.

Supporting Information Available: TEM characterization, SEM characterization, characterization of CdS and CdS/CdSe sensitized electrodes, cyclic voltammetry, and cell reproducibility. This material is available free of charge via the Internet at http:// pubs.acs.org.

\section{REFERENCES AND NOTES}

1. Itzhaik, Y.; Niitsoo, O.; Page, M.; Hodes, G. $\mathrm{Sb}_{2} \mathrm{~S}_{3}$-Sensitized Nanoporous $\mathrm{TiO}_{2}$ Solar Cells. J. Phys. Chem. C 2009, 113, 4254-4256.

2. Lévy-Clément, C.; Tena-Zaera, R.; Ryan, M. A.; Katty, A. Hodes, G. CdSe Sensitized p-CuSCN/Nanowire n-ZnO Heterojunctions. Adv. Mater. 2005, 17, 1512-1515.

3. O' Regan, B.; Grätzel, M. A Low-Cost High-Efficiency Solar Cell Based on Dye-Sensitized Colloidal $\mathrm{TiO}_{2}$ Films. Nature 1991, 353, 737.

4. Kamat, P. V. Quantum Dot Solar Cells. Semiconductor Nanocrystals as Light Harvesters. J. Phys. Chem. C 2008, 112, 18737-18753.

5. Yu, W.; Qu, L. H.; Guo, W. Z.; Peng, X. G. Experimental Determination of the Extinction Coefficient of CdTe, CdSe, and CdS Nanocrystals. Chem. Mater. 2003, 15, 2854-2860.

6. Vogel, R.; Hoyer, P.; Weller, H. Quantum-Sized PdS, CdS, $\mathrm{Ag}_{2} \mathrm{~S}, \mathrm{Sb}_{2} \mathrm{~S}_{3}$ and $\mathrm{Bi}_{2} \mathrm{~S}_{3}$ Particles as Sensitizers for Various Nanoporous Wide-Bandgap Semiconductors. J. Phys. Chem. B 1994, 98, 3183-3188.

7. Vogel, R.; Pohl, K.; Weller, H. Sensitization of Highly Porous, Polycrystalline $\mathrm{TiO}_{2}$ Electrodes by Quantum Sized CdS. Chem. Phys. Lett. 1990, 174 (3-4), 241-246.

8. Buhbut, S.; Itzhakov, S.; Tauber, E.; Shalom, M.; Hod, I.; Geiger, T.; Garini, Y.; Oron, D.; Zaban, A. Built-in Quantum Dot Antennas in Dye-Sensitized Solar Cells. ACS Nano 2010, 4, 1293-1298.

9. Shalom, M.; Albero, J.; Tachan, Z.; Martínez-Ferrero, E.; Zaban, A.; Palomares, E. Quantum Dot-Dye BilayerSensitized Solar Cells: Breaking the Limits Imposed by the Low Absorbance of Dye Monolayers. J. Phys. Chem. Lett. 2010, 1, 1134-1138.

10. Mora-Seró, I.; Gross, D.; Mittereder, T.; Lutich, A. A.; Susha, A.; Dittrich, T.; Belaidi, A.; Caballero, R.; Langa, F.; Bisquert, J.; et al. Nanoscale Interaction between CdSe or CdTe Nanocrystals and Molecular Dyes Fostering or Hindering Directional Charge Separation. Small 2010, 6, 221-225.

11. Mora-Seró, I.; Likodimos, V.; Giménez, S.; Martínez-Ferrero, E.; Albero, J.; Palomares, E.; Kontos, A. G.; Falaras, P.; Bisquert, J. Fast Regeneration of CdSe Quantum Dots by Ru Dye in Sensitized $\mathrm{TiO}_{2}$ Electrodes. J. Phys. Chem. C 2010, 114, 6755-6761.

12. Fan, S.-Q.; Fang, B.; Kim, J. H.; Kim, J.-J.; Yu, J.-S.; Ko, J. Hierarchical Nanostructured Spherical Carbon with Hollow Core/Mesoporous Shell as a Highly Efficient Counter Electrode in CdSe Quantum-Dot Sensitized Solar Cells. Appl. Phys. Lett. 2010, 96, 063501.

13. Lee, Y.-L.; Lo, Y.-S. Adv. Funct. Mater. 2009, 19, 604, presents an efficiency of $4.2 \%$ but with a discrepancy between photocurrent and IPCE data.

14. Mora-Seró, I.; Giménez, S.; Fabregat-Santiago, F.; Gómez, R.; Shen, Q.; Toyoda, T.; Bisquert, J. Recombination in Quantum Dot Sensitized Solar Cells. Acc. Chem. Res. 2009, 42, 1848-1857.

15. Hodes, G. Comparison of Dye- and SemiconductorSensitized Porous Nanocrystalline Liquid Junction Solar Cells. J. Phys. Chem. C 2008, 112, 17778-17787.

16. Lee, H. J.; Wang, M.; Chen, P.; Gamelin, D. R.; Zakeeruddin, S. M.; Grätzel, M.; Nazeeruddin, M. K. Efficient CdSe Quantum Dot-Sensitized Solar Cells Prepared by an Improved Successive Ionic Layer Adsorption and Reaction Process. Nano Lett. 2009, 9, 4221-4227.

17. Robel, I.; Subramanian, V.; Kuno, M.; Kamat, P. V. Quantum Dot Solar Cells. Harvesting Light Energy with CdSe Nanocrystals Molecularly Linked to Mesoscopic TiO, Films. J. Am. Chem. Soc. 2006, 128, 2385-2393.

18. Giménez, S.; Mora-Seró, I.; Macor, L.; Guijarro, N.; LanaVillarreal, T.; Gómez, R.; Diguna, L. J.; Shen, Q.; Toyoda, T.; Bisquert, J. Improving the Performance of Colloidal Quantum Dot Sensitized Solar Cells. Nanotechnology 2009, 20, 295204

19. Shen, Q.; Kobayashi, J.; Diguna, L. J.; Toyoda, T. Effect of ZnS Coating on the Photovoltaic Properties of CdSe Quantum Dot-Sensitized Solar Cells. J. Appl. Phys. 2008, $103,084304$. 

Hodes, G. Chemical Bath Deposited CdS/CdSe-Sensitized Porous $\mathrm{TiO}_{2}$ Solar Cells. J. Photochem. Photobiol., A 2006, 181, 306-311.

21. Hodes, G.; Manassen, J.; Cahen, D. Electrocatalytic Electrodes for the Polysulfide Redox System. J. Electrochem. Soc. 1980, 127, 544-549.

22. Fabregat-Santiago, F.; Bisquert, J.; Garcia-Belmonte, G.; Boschloo, G.; Hagfeldt, A. Influence of Electrolyte in Transport and Recombination in Dye-Sensitized Solar Cells Studied by Impedance Spectroscopy. Sol. Energy Mater. Sol. Cells 2005, 87, 117-131.

23. Bisquert, J. Theory of the Impedance of Electron Diffusion and Recombination in a Thin Layer. J. Phys. Chem. B 2002, 106, 325-333.

24. Bisquert, J. Chemical Capacitance of Nanostructured Semiconductors: Its Origin and Significance for Nanocomposite Solar Cells. Phys. Chem. Chem. Phys. 2003, 5, 5360-5364.

25. Barea, E. M.; Shalom, M.; Giménez, S.; Hod, I.; Mora-Seró, l.; Zaban, A.; Bisquert, J. Design of Injection and Recombination in Quantum Dot Sensitized Solar Cells. J. Am. Chem. Soc. 2010, 132, 6834-6839.

26. Impedance Spectroscopy Test. www.istest.eu.

27. Bisquert, J.; Mora-Seró, I. Simulation of Steady-State Characteristics of Dye-Sensitized Solar Cells and the Interpretation of the Diffusion Length. J. Phys. Chem. Lett. 2010, 1, 450-456.

28. Bisquert, J.; Fabregat-Santiago, F.; Mora-Seró, I.; GarciaBelmonte, G.; Giménez, S. Electron Lifetime in DyeSensitized Solar Cells: Theory and Interpretation of Measurements. J. Phys. Chem. C 2009, 113, 17278-17290.

29. Bisquert, J.; Zaban, A.; Greenshtein, M.; Mora-Seró, I. Determination of Rate Constants for Charge Transfer and the Distribution of Semiconductor and Electrolyte Electronic Energy Levels in Dye-Sensitized Solar Cells by Open-Circuit Photovoltage Decay Method. J. Am. Chem. Soc. 2004, 126, 13550-13559.

30. Lee, Y.-L.; Lo, Y.-S. Highly Efficient Quantum-Dot-Sensitized Solar Cell Based on Co-Sensitization of CdS/CdSe. Adv. Funct. Mater. 2009, 19, 604-609. 